\title{
A Review on Differential Lines and Its Study Based On Differential S Parameters
}

\author{
Ashish Lohana ${ }^{1}$, Sulabha Ranade ${ }^{2}$, Jyoti Varavadekar ${ }^{3}$ \\ ${ }^{1,3}$ (EXTC, K.J. Somaiya CoE / University of Mumbai, India), ${ }^{2}$ (SAMEER, IIT Campus, Powai, India)
}

\begin{abstract}
In this paper a brief review of differential signalling is given and it is studied why using single ended standard S parameters does not give us a complete understanding of a Differential line and thus comes a new mixed mode S parameters that helps us understand the common mode to differential mode conversion and vice versa, in a simplified manner. Also conversion method from single ended S parameters to Approximate and True Mixed mode S parameters is studied in brief and derived.
\end{abstract}

Keywords - Differential port, Differential signaling, Even and Odd mode Impedance, Mode conversion, Single ended $S$ parameters.

\section{Introduction}

All of us do use single ended signaling in our day to day life. The normal electrical or electronic appliances that we use in our daily practices are all single ended; they all have a single wire carrying all the voltages and a current called as 'main' and another which is used for reference called as 'neutral' or 'ground'. Thus Single Ended connection is an electrical connection where one wire carries the signal and another wire is connected to reference, mostly ground. Thus the measured input and output signal is the difference between the signal and reference ground, thus source current is equal to return current.

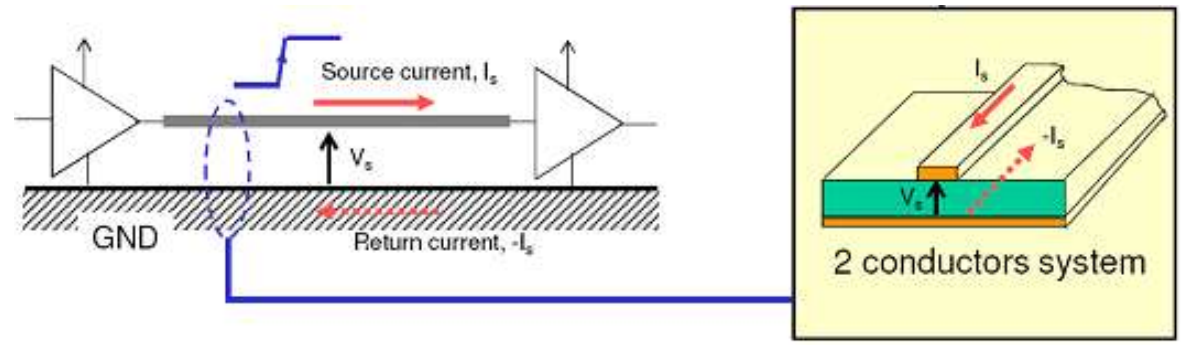

Fig. 1 Single ended signalling

The ground is considered to be at a constant $0 \mathrm{~V}$, but in reality the ground, or earth, is at a different level in different places. Making a connection between two grounds at different levels can drive large currents, known as earth or ground loops. This can lead to errors when using single-ended inputs [1]. Also the errors are caused as a result of; all the signal wires act as ariels i.e. they pick up surrounding electrical activity that gets induced as noise. Noise being additive in nature, one cannot distinguish between the signal and noise. Also when transmitting high-speed electrical signal, the EM fields for the signal trace and the return current on the ground plane have the potential to cause electrical interference on adjacent circuits. Furthermore with digital system going for lower operating voltage, logic signal swing and noise margin also decrease, this undermines the noise immunity of the digital system.

Differential signaling was proposed to eliminate the problems associated with single ended signals. A brief idea on differential signaling is presented and then we will study how are differential lines studied using Mixed mode $\mathrm{S}$ parameters and how to obtain approximate and True S parameters from single ended standard S parameters.

\section{Differential Lines}

Differential connection is an electrical connection using two wires, one of which carries the normal signal $(\mathrm{V})$ and the other carries an inverted version the signal (-V). Since a pair of lines are required for differential signaling, microstrip coupled lines are used for high speed data transmission at microwave frequencies excited differentially. 


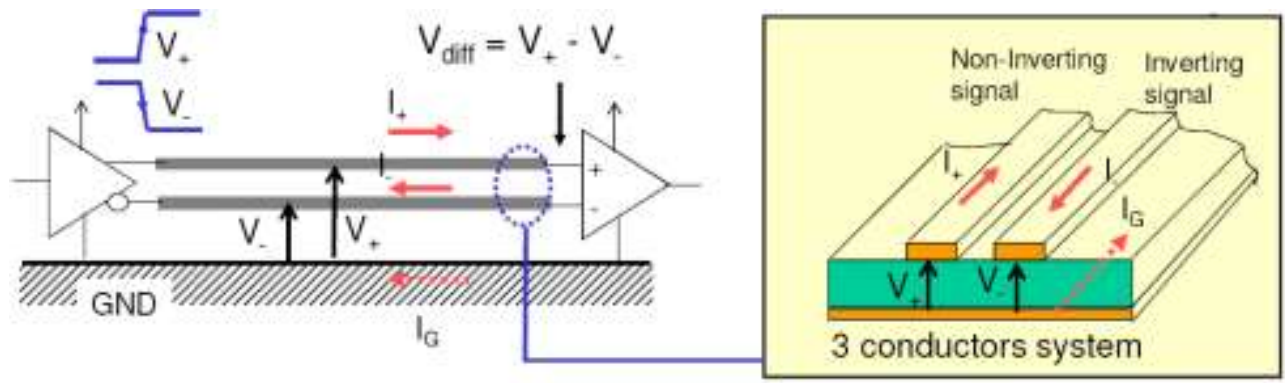

Fig. 2 Differential Signalling [1]

The differential amplifier at the receiver subtracts the inverted version from the normal signal to yields a signal twice of original signal:

Where ' $n$ ' is the additive noise.

$$
(\mathrm{V}+\mathrm{n})-(-\mathrm{V}+\mathrm{n})=2 \mathrm{~V}
$$

This subtraction is intended to cancel out any noise induced in the wires, on the assumption that the same level of noise will have been induced in both wires.

Also:

By design is called as balanced signal therefore $\mathrm{I}_{\mathrm{G}}=0$

$$
\left|I_{+}\right|=\left|I_{-}\right|
$$

Usually the signal conductors are close together, forming a tightly coupled system, resulting in $\mathrm{I}_{\mathrm{G}}=0$ (balanced condition).

For the currents to be equal and opposite, as needed for balance, the following features must exist:

1. The amplitude in both circuits must be identical

2. The load impedance must be identical

3. There can be no skew between rising and falling edges

4. The rise and fall time must be identical

5. The physical trace routes must be not only equaled in length overall, but also balanced along their entire length

\section{Coupling to any other conductors must be equal [2]}

Advantage of differential signaling is, it uses lower voltage levels than single ended signals because the threshold in differential receiver is better controlled than in single ended due to high noise immunity. The lower voltage swing leads to faster circuits and reduction in power consumption, thereby increasing the bandwidth.

Wherever there is a differential signal, there will also be a common mode signal. LVDS (low voltage differential signaling) signaling, for example, uses a $400 \mathrm{mV}$ differential signal centered at $1.2 \mathrm{~V}$.

From Fig. 2 the differential and common mode voltages, currents and impedances can be expressed as [3]:

$$
\begin{gathered}
\mathrm{V}_{\text {diff }}=\mathrm{Vd}=\left(\mathrm{V}_{+}-\mathrm{V}_{-}\right) \\
\mathrm{V}_{\text {common }}=\mathrm{Vc}=\left(\mathrm{V}_{+}+\mathrm{V}_{-}\right) / 2 \\
\mathrm{I}_{\text {diff }}=\mathrm{Id}=\left(\mathrm{I}_{+}-\mathrm{I}_{-}\right) / 2 \\
\mathrm{I}_{\text {common }}=\mathrm{Ic}=\left(\mathrm{I}_{+}+\mathrm{I}_{-}\right) \\
\mathrm{Z}_{\text {diff }}=\mathrm{Z}_{\mathrm{d}}=\mathrm{Vd} / \mathrm{Id}=2 \mathrm{Zoo} \\
\mathrm{Z}_{\text {comman }}=\mathrm{Z}_{\mathrm{c}}=\mathrm{Vc} / \mathrm{Ic}=\mathrm{Zoe} / 2
\end{gathered}
$$

Where Zoe - even mode impedance, Zoo - odd mode impedance.

A pair of transmission lines, each with impedance $Z_{0}$, will have different impedance for differential signals and for common mode signals. The differential impedance will depend on the spacing of the lines. If the lines are far apart (spacing > width), then Zdiff $=2 * Z 0$. As the lines are brought closer together, the coupling between the traces increases and Zdiff decreases. Both the differential signal and the common mode signal will travel down interconnects and suffer from reflections at impedance mismatches. The differential and common mode signals will behave differently, since they will see different effective impedances and travel speeds down the transmission lines [3].

\section{Use of S parameters to study Signals}

$S$ parameters are normally used when one needs to study the electrical behaviour of a circuit at high frequencies. These S parameters give us the incident power, reflected power and reflection coefficients (amount 
of reflections). Traditional S-parameters called single ended S parameters are useful when working with single ended devices. But mixed-mode S-parameters provide the capability of analyzing and visualizing the signal flow through differential (balanced) lines and devices found in modern high-speed digital communications systems. Using Single ended S parameters for differentially excited coupled lines, does not provide much of the insight to differential (or common-mode) operation because each port contains the differential and common mode response. To overcome this problem, a system similar to that used to describe the four transfer gains $\left(\mathrm{A}_{\mathrm{cc}}, \mathrm{A}_{\mathrm{dd}}\right.$, $\mathrm{A}_{\mathrm{cd}}$, and $\mathrm{A}_{\mathrm{dc}}$ ) introduced by Middlebrook3) is used. This system of S-parameters (known as mixed mode $\mathrm{S}$ parameters) was introduced by Bockelman.

\section{Conversion of Single ended mode S parameters to Approximate Mixed mode S parameters}

Conversion of Single ended mode $\mathrm{S}$ parameters to mixed mode $\mathrm{S}$ parameters begins by grouping ports one and two together (to form a differential port one) and grouping ports three and four together (to form a differential port two). This grouping is shown in the Fig. 3

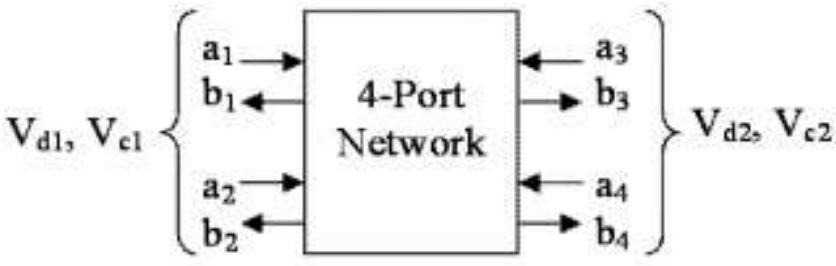

Fig. 3 Port configuration

With this conversion between single ended voltages and currents to differential (and common-mode) voltages and currents, a way to convert from single-ended S-parameters to mixed mode S-parameters was found. it is now convenient to define the mixed-mode S-parameters, using the definition for the incident and returning waves, differential and common-mode incident and returning power wave can be defined as [4]:

$$
\begin{aligned}
& \mathrm{a}_{\mathrm{dn}}=\left(\mathrm{V}_{\mathrm{dn}}+\mathrm{I}_{\mathrm{dn}} \mathrm{Z}_{\mathrm{dn}}\right) /\left(2 \operatorname{sqrt}\left(\mathrm{Z}_{0}\right)\right) \\
& \mathrm{b}_{\mathrm{dn}}=\left(\mathrm{V}_{\mathrm{dn}}-\mathrm{I}_{\mathrm{dn}} \mathrm{Z}_{\mathrm{dn}}\right) /\left(2 \operatorname{sqrt}\left(\mathrm{Z}_{0}\right)\right) \\
& \mathrm{a}_{\mathrm{cn}}=\left(\mathrm{V}_{\mathrm{cn}}+\mathrm{I}_{\mathrm{cn}} \mathrm{Z}_{\mathrm{dn}}\right) /\left(2 \operatorname{sqrt}\left(\mathrm{Z}_{0}\right)\right) \\
& \mathrm{b}_{\mathrm{cn}}=\left(\mathrm{V}_{\mathrm{cn}}-\mathrm{I}_{\mathrm{cn}} \mathrm{Z}_{\mathrm{dn}}\right) /\left(2 \operatorname{sqrt}\left(\mathrm{Z}_{0}\right)\right)
\end{aligned}
$$

$\mathrm{a}_{\mathrm{dn}}=$ differential mode incident power

$\mathrm{a}_{\mathrm{cn}}=$ common mode incident power

$b_{\mathrm{dn}}=$ differential mode reflected power

$\mathrm{b}_{\mathrm{cn}}=$ common mode reflected power

$\mathrm{V}_{\mathrm{dn}}=$ the differential voltage at port $\mathrm{n}$,

$\mathrm{V}_{\mathrm{cn}}=$ the common-mode voltage at port $\mathrm{n}$,

$\mathrm{I}_{\mathrm{dn}}=$ the differential current at port $\mathrm{n}$,

$\mathrm{I}_{\mathrm{dc}}=$ the common-mode current at port $\mathrm{n}$,

$\mathrm{Z}_{\mathrm{dn}}=$ the differential-mode characteristic impedance at port $\mathrm{n}$, and

$\mathrm{Z}_{\mathrm{cn}}=$ the common-mode characteristic impedance at port $\mathrm{n}$.

With the definition of the power waves in 9 through 12, mixed-mode S-parameters can be defined as:

$$
\begin{aligned}
& b_{d 1}=S_{d d 11} a_{d 1}+S_{d d 12} a_{d 2}+S_{d c 11} a_{c 1}+S_{d c 12} a_{c 2} \\
& b_{d 2}=S_{d d 21} a_{d 1}+S_{d d 22} a_{d 2}+S_{d c 21} a_{c 1}+S_{d c 22} a_{c 2} \\
& b_{c 1}=S_{c d 11} a_{d 1}+S_{c d 12} a_{d 2}+S_{c c 11} a_{c 1}+S_{c c 12} a_{c 2} \\
& b_{c 2}=S_{c d 11} a_{d 1}+S_{c d 12} a_{d 2}+S_{c c 21} a_{c 1}+S_{c c 22} a_{c 2}
\end{aligned}
$$

Where Smmij $=\mathrm{S}$ (output mode) (input mode) (output port) (input port)

The above equations can be represented in matrix form as: 


$$
\left(\begin{array}{c}
b_{d 1} \\
b_{d 2} \\
b_{c 1} \\
b_{c 2}
\end{array}\right)=\left(\begin{array}{cccc}
S_{d d 11} & S_{d d 12} & S_{c d 11} & S_{c d 12} \\
S_{d d 21} & S_{d d 22} & S_{c d 21} & S_{c d 22} \\
S_{d c 11} & S_{d c 12} & S_{c c 11} & S_{c c 12} \\
S_{d c 21} & S_{d c 22} & S_{c c 21} & S_{c c 22}
\end{array}\right)\left(\begin{array}{c}
a_{d 1} \\
a_{d 2} \\
a_{c 1} \\
a_{c 2}
\end{array}\right)
$$

Where: $S_{\mathrm{dd}}=$ the differential S-parameters,

$\mathrm{S}_{\mathrm{cc}}=$ the common-mode S-parameters,

$\mathrm{S}_{\mathrm{dc}}=$ the mode conversion that occurs when the device is excited with common mode signal and the differential signal is measured, and

$\mathrm{S}_{\mathrm{cd}}=$ the mode conversion that occurs when the device is excited with a differential- mode signal and the common mode response is measured.

To convert from single-ended S parameters to mixed-mode S-parameters, it is assumed that the DUT is being fed from differential input lines and that $\mathrm{Zoe}=\mathrm{Zoo}=\mathrm{Z}$. The assumption of differential input lines is not limiting, since it is possible to define the length of the lines to be arbitrarily small. The assumption of Zoe $=$ Zoo $=\mathrm{Z} 0$ implies that the differential input lines are not coupled [4].

Taking the definitions of $\mathrm{Vd}, \mathrm{Vc}$, Id, and Ic from Equations. 3 through 6 and plugging them into Eqs. 9 through 12 , and taking $\mathrm{Zd}$ to be $2 \mathrm{Z0}$, the following equations are the result:

$$
\left.\begin{array}{rl}
\mathrm{a}_{\mathrm{d} 1} & =\left(\mathrm{a}_{1}-\mathrm{a}_{2}\right) / \operatorname{sqrt}(2) \\
\mathrm{a}_{\mathrm{c} 1} & =\left(\mathrm{a}_{1}+\mathrm{a}_{2}\right) / \operatorname{sqrt}(2) \\
\mathrm{b}_{\mathrm{d} 1} & =\left(\mathrm{b}_{1}-\mathrm{b}_{2}\right) / \text { sqrt (2) } \\
\mathrm{b}_{\mathrm{c} 1} & =\left(\mathrm{b}_{1}+\mathrm{b}_{2}\right) / \text { sqrt (2) } \\
\mathrm{a}_{\mathrm{d} 2} & =\left(\mathrm{a}_{3}-\mathrm{a}_{4}\right) / \text { sqrt (2) } \\
\mathrm{a}_{\mathrm{c} 2}=\left(\mathrm{a}_{3}+\mathrm{a}_{4}\right) / \operatorname{sqrt}(2) \\
\mathrm{b}_{\mathrm{d} 2}=\left(\mathrm{b}_{3}-\mathrm{b}_{4}\right) / \operatorname{sqrt}(2) \\
\mathrm{b}_{\mathrm{c} 2}=\left(\mathrm{b}_{3}+\mathrm{b}_{4}\right) / \operatorname{sqrt}(2)
\end{array}\right\}
$$

Representing the above in matrix form

$$
\begin{aligned}
& \left(\begin{array}{l}
\mathrm{ad} 1 \\
\mathrm{ad} 2 \\
\mathrm{ac} 1 \\
\mathrm{ac} 2
\end{array}\right)=1 / \operatorname{Sqrt}(2)\left(\begin{array}{cccc}
1 & -1 & 0 & 0 \\
0 & 0 & 1 & -1 \\
1 & 1 & 0 & 0 \\
0 & 0 & 1 & 1
\end{array}\right)\left(\begin{array}{c}
\mathrm{ad} 1 \\
\mathrm{ad} 2 \\
\mathrm{ac} 1 \\
\mathrm{ac} 2
\end{array}\right) \\
& \text { therefore: } \mathrm{a}^{\mathrm{mm}}=\mathrm{M} \mathrm{a}^{\text {std }} \text { and } \mathrm{b}^{\mathrm{mm}}=\mathrm{Mb}^{\text {std }} \\
& \text { Where } M=1 / \text { Sqrt (2) }\left(\begin{array}{cccc}
1 & -1 & 0 & 0 \\
0 & 0 & 1 & -1 \\
1 & 1 & 0 & 0 \\
0 & 0 & 1 & 1
\end{array}\right)
\end{aligned}
$$

Thus mixed Mode $\mathrm{S}$ parameters can be given as:

$$
\mathrm{S}^{\mathrm{mm}}=\mathrm{M} \mathrm{S}^{\mathrm{std}} \mathrm{M}-1
$$

The superscript "mm" represents mixed mode and "std" represents the standard single ended S parameters. 


\section{Simulations}

The simulations were done to get the mixed mode s parameters from standard s parameters. For simulation a symmetric microstrip coupled line as shown in Fig. 4, was selected with the following parameters as in Table 1.

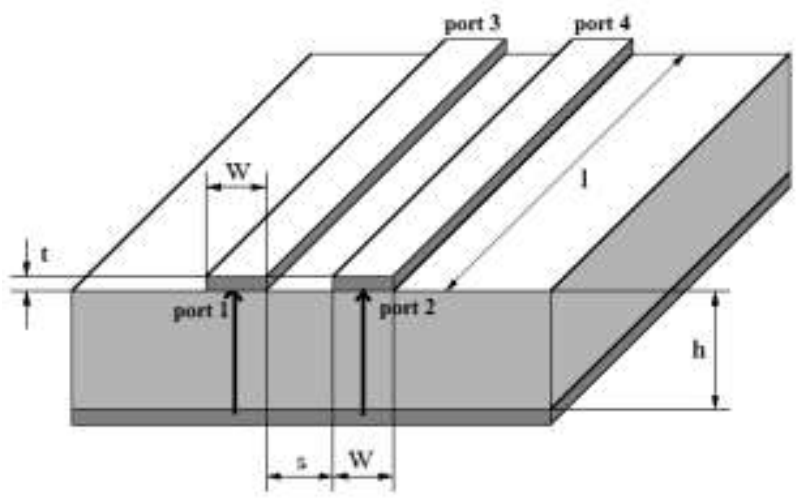

Fig. 4 Symmetric Microstrip Coupled line

TABLE. 1 Parameters Considered During Simulation

\begin{tabular}{|l|c|c|}
\hline \multicolumn{1}{|c|}{ Parameters } & Values Simulation 1 & Values Simulation 2 \\
\hline Dielectric constant & 10.0 & 10.0 \\
\hline Substrate thickness (h) & $1.70 \mathrm{~mm}$ & $1.70 \mathrm{~mm}$ \\
\hline Conductor thickness(t) & $0.01 \mathrm{~mm}$ & $0.01 \mathrm{~mm}$ \\
\hline Conductor Width(W) & $1.75 \mathrm{~mm}$ & $1.75 \mathrm{~mm}$ \\
\hline Spacing between conductor(s) & $0.25 \mathrm{~mm}$ & $0.75 \mathrm{~mm}$ \\
\hline Dielectric loss Tangent & 0.001 & 0.001 \\
\hline Conductor length(l) & $50 \mathrm{~mm}$ & $50 \mathrm{~mm}$ \\
\hline $\mathrm{Z}_{\mathrm{oo}}$ & 31.253 & 40.52 \\
\hline $\mathrm{Z}_{\mathrm{oe}}$ & 84.20 & 78.79 \\
\hline $\mathrm{Z}_{0}$ & 51.23 & 56.50 \\
\hline
\end{tabular}

VI. Simulation Results

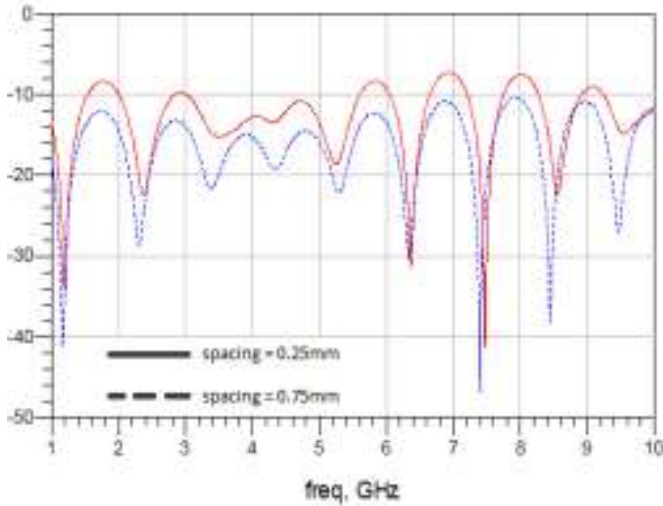

Fig. 5b S14 magnitude dB

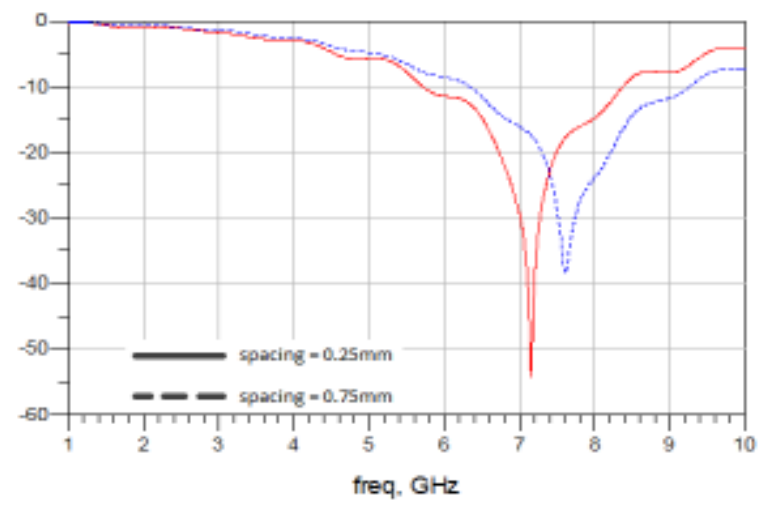

Fig. 5a S12 magnitude dB

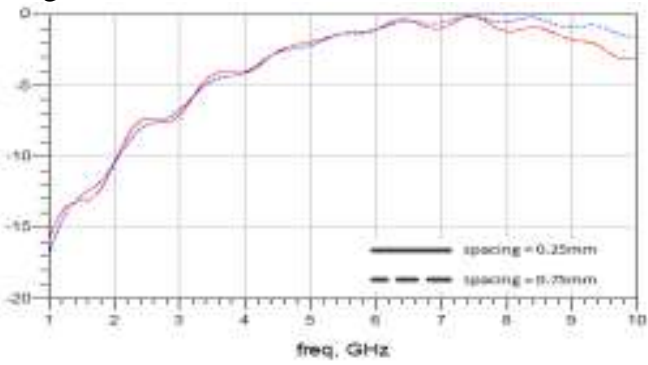

Fig. 5c S13 magnitude dB 
From the fig. 5a and fig. $5 \mathrm{c}$ it is seen that near end and far end cross talk is high. From fig. 5b we can say that transmission loss is less. But the above results don't give us the conversion of common to differential mode or differential to common mode conversion.

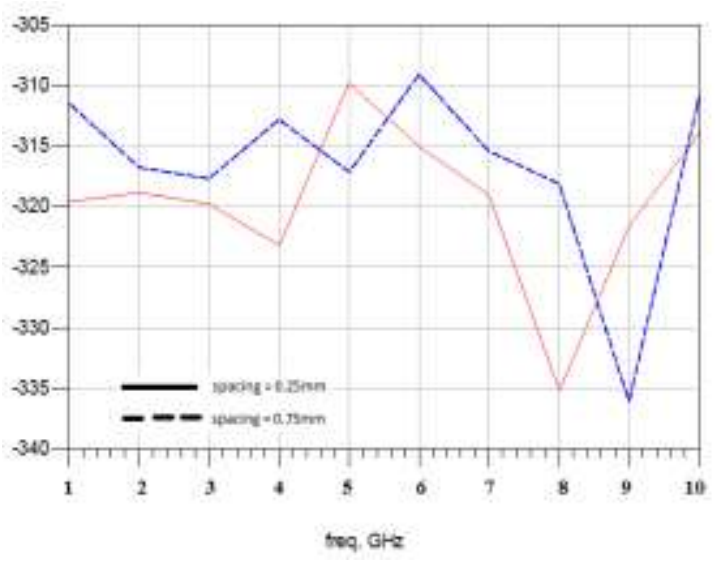

Fig. 6a Scd12 magnitude dB

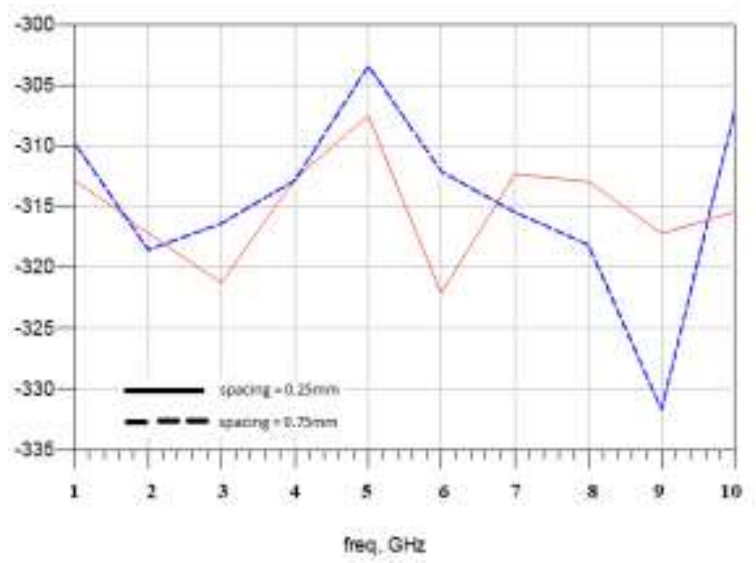

Fig. 6b Sdc12 magnitude dB

From figure $6 \mathrm{a}$ and $6 \mathrm{~b}$ it is seen that common mode to differential mode and differential to common mode conversion is very less; but as we increase the spacing between the two lines the mode conversion increases, as seen conversion from common mode to differential mode is less for lines with spacing of $0.25 \mathrm{~mm}$ and is increased for $0.75 \mathrm{~mm}$.

\section{Conversion Of Single Mode S Parameters To Mixed Mode S Parameters Considering Coupling Effect (True S Parameters)}

What we saw in the earlier section was conversion of single ended s parameters to mixed mode $\mathrm{s}$ parameters, assuming that even, odd and characteristic impedance are all equal, thus there is no coupling between the two lines. But practically even, odd and characteristic impedance are never the same due to coupling between the two lines, which must be there for benefit of noise removal, crosstalk reduction and EMI reduction [5]. Considering the above scenario the earlier section equations are no more valid and give us approximate mixed mode $\mathrm{S}$ parameters.

A scheme was introduced to convert single ended $S$ parameters to mixed mode $S$ parameters by introducing two constants Koo and Koe that depend on the amount of coupling between the lines [6]:

$$
\mathrm{KoO}=\mathrm{Zoo} / \mathrm{Z0} \text { and } \mathrm{Koe}=\mathrm{Zoe} / \mathrm{Z0}
$$

If there is no coupling between the lines then $\mathrm{Zoo}=\mathrm{Zoe}=\mathrm{Z} 0$ and thus $\mathrm{Koo}=\mathrm{Koe}=1$.

From equations 7,8 and 18 we have:

$$
\begin{gathered}
Z d / 2=Z o o=\text { Koo Z0 } \\
2 \text { Zc }=\text { Zoe }=\text { Koe Z0 }
\end{gathered}
$$

Substituting equations 3 to 8 and 19 in equations 9 to 12 we get a bunch of equations that can be represented as:

$$
\begin{gathered}
\mathrm{a}^{\text {true } \mathrm{mm}}=\mathrm{M} 1 \mathrm{a}^{\text {std }}+\mathrm{M} 2 \mathrm{~b}^{\text {std }} \quad \text { and } \quad \mathrm{b}^{\text {true mm }}=\mathrm{M} 1 \mathrm{~b}^{\text {std }}+\mathrm{M} 2 \mathrm{a}^{\text {std }} \\
\text { thus }\left[\mathrm{S}^{\text {true mm }}\right]=\left\{[\mathrm{M} 1]\left[\mathrm{S}^{\text {std }}\right]+[\mathrm{M} 2]\right\}\left\{[\mathrm{M} 1]+[\mathrm{M} 2]\left[\mathrm{S}^{\text {std }}\right]\right\}^{-1}
\end{gathered}
$$

$\mathrm{M} 1=\left[\begin{array}{cccc}\mathrm{e} & -\mathrm{e} & 0 & 0 \\ 0 & 0 & \mathrm{e} & -\mathrm{e} \\ \mathrm{f} & \mathrm{f} & 0 & 0 \\ 0 & 0 & \mathrm{f} & \mathrm{f}\end{array}\right] \quad \mathrm{M} 2=\left[\begin{array}{cccc}\mathrm{g} & -\mathrm{g} & 0 & 0 \\ 0 & 0 & \mathrm{~g} & -\mathrm{g} \\ \mathrm{h} & \mathrm{h} & 0 & 0 \\ 0 & 0 & \mathrm{~h} & \mathrm{~h}\end{array}\right]$

$\mathrm{e}=(1+\mathrm{Koo}) /(2 \operatorname{sqrt}(2 \mathrm{Koo}))$

$\mathrm{f}=(1+$ Koe $) /(2 \operatorname{sqrt}(2$ Koe $))$ 
$\mathrm{g}=(1-\mathrm{Koo}) /(2 \operatorname{sqrt}(2 \mathrm{Koo}))$

$\mathrm{h}=(1-$ Koe $) /(2 \operatorname{sqrt}(2$ Koe $))$

When $\mathrm{Zoo}=\mathrm{Zoe}=\mathrm{Z} 0$ then $\mathrm{Koo}=\mathrm{Koe}=1$ thus M2 $=[0]$ and M1 reduces to $\mathrm{M}$ and thus $\mathrm{S}^{\text {true } \mathrm{mm}}=$ $\mathrm{S}^{\mathrm{mm}}$

The following results are for the same values of microstrip as in Table 1. Simulation 2:

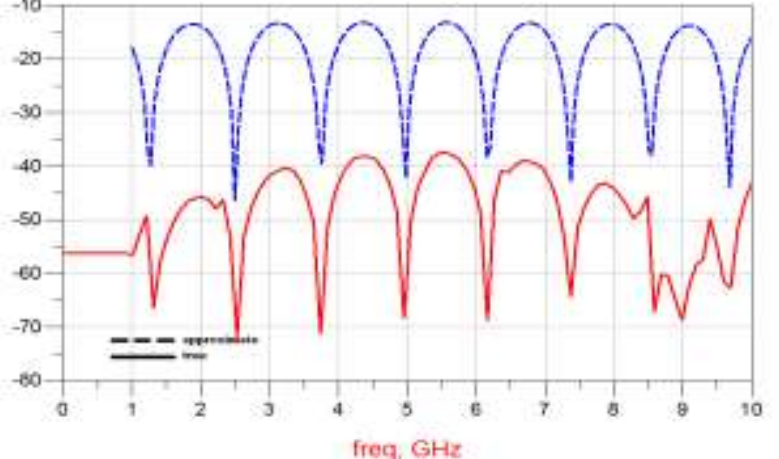

Fig. 7a Sdd11 magnitude $d B$

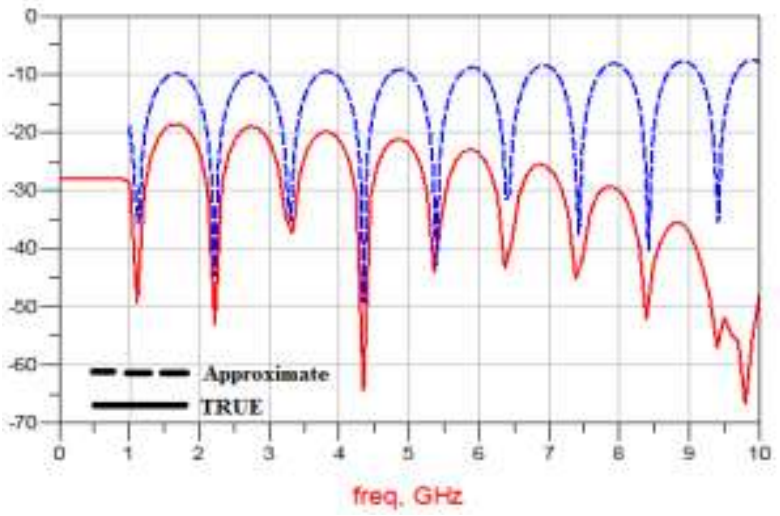

Fig. 7c Scc11 magnitude dB

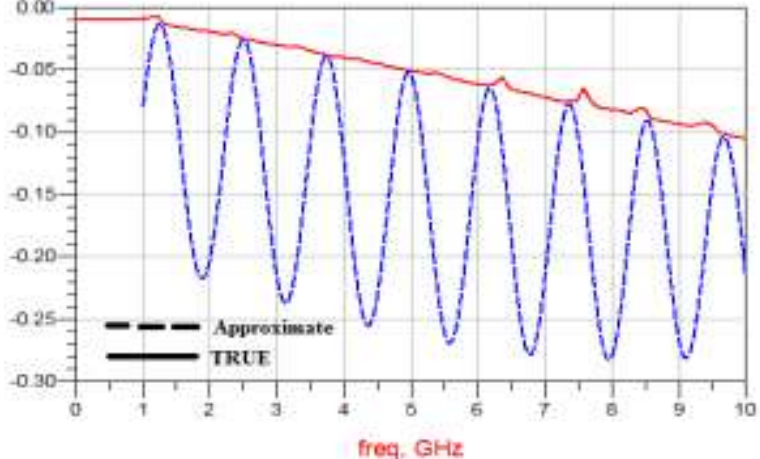

Fig. 7a Sdd21 magnitude $d B$

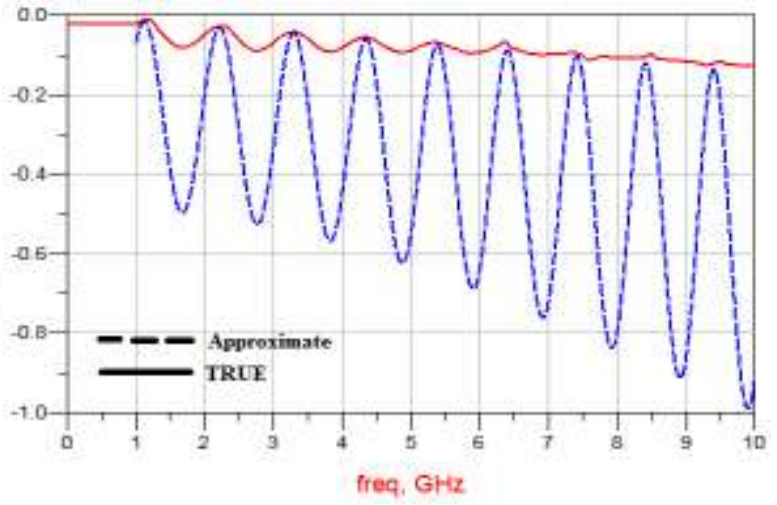

Fig. 7d Scc21 magnitude dB

\section{Conclusion}

The review on differential lines shows clearly its advantages of low interference, less cross talk, reduction in transmission power over single line signaling. As seen in simulation examples, the mixed mode $\mathrm{S}$ parameters gave us clear information about the conversion from common mode to differential mode and vice versa, which lacked in single mode $\mathrm{S}$ parameters. Also for reduced mode conversion and low external interference the two lines must be tightly coupled. Assumption of non coupling differential lines led us to approximate mixed mode $\mathrm{S}$ parameters that are easy to calculate from single mode $\mathrm{S}$ parameters but not accurate. The accuracy can be obtained by considering the coupling effect by introduction of constants Koo and Koe that depend on the amount of coupling between lines.

\section{Books:}

\section{References}

[1] H. Johnson, M. Graham, High-speed signal propagation Advanced black magic Prentice-Hall, 2002.

\section{Journal Papers:}

[2] Bruce Archambeault, Associate Editor, Member IEEE, Design Tip - EMC Considerations for Differential (Balanced) Lines

[3] David E. Bockelman, Member, IEEE, and William R. Eisenstadt, Senior Member, IEEE, Pure-Mode Network Analyzer for OnWafer, Measurements of Mixed-Mode S-Parameters of Differential Circuits, Ieee Transactions On Microwave Theory And Techniques, Vol. 45, No. 7, July 1997.

[4] Garth Sundberg, Member of the Technical Staff Technology, Research, and Development, Grasp The Meaning Of Mixed- Mode S-Parameters. Microwaves and RF, vol. 40, pp. 99-104, May 2001.

[5] Wolfgang Maichen and Bo Krsnik, A Practical Guide to Lossy Differential Lines, Teradyne, Inc.

\section{Proceedings Papers:}

[6] Allan Huynh, Pär Håkansson and Shaofang Gong, Mixed-Mode S-Parameter Conversion for Networks with Coupled Differential Signals Department of Science and Technology, Linköping University Norrköping, Bredgatan 33, SE-601 74 Norrköping, Sweden. 